\title{
Is now the time to extend biomarker stratification to other RAS-family genes?
}

A new study published in The New England Journal of Medicine has shown that patients without the common KRAS exon 2 mutations, but with other RAS mutations, have poorer survival when given anti-EGFR therapy in combination with chemotherapy than those with just the common exon 2 KRAS mutations. These results highlight the possible importance of genetic features other than KRAS exon 2 in patients with metastatic colorectal cancer.

EGFR (also known as HER-1) is an important component of cell signalling that includes the Ras/Raf/MAPK and PI3K/Akt pathways, which influence cell-cycle progression, angiogenesis, cell growth and survival. These pathways are implicated in numerous cancer types and targeted therapies against various points of the pathways have been developed and are used clinically.

Several clinical trials have shown that patients with activating mutations of exon 2 of KRAS do not benefit from antiEGFR antibody therapy. The evidence is robust enough that standard testing of KRAS status is performed in clinical trials in patients with colorectal cancer, of whom approximately $40 \%$ harbour mutations at this locus, and these genetic tests inform treatment options for affected patients. However, until now it was unclear what clinical effect other RAS mutations - such as mutations in KRAS exons 3 and 4, and NRAS exons 2, 3 and 4-had for treatment.

"In the initial 2010 report, the PRIME clinical trial demonstrated that the addition of panitumumab, which targets EGFR, to oxaliplatin, fluorouracil and leucovorin (FOLFOX4) in patients with metastatic colorectal cancer improved progression-free survival," explains lead investigator Jean-Yves Douillard. "However, the benefit was restricted to patients without KRAS mutations. By contrast, patients with mutations experienced a detrimental effect. The present study extended the search for mutations to other members of the RAS family among patients initially found to be nonmutant for KRAS exon 2."

Overall, $90 \%$ of the patients who underwent random assignment had their RAS status ascertained. A total of 512 patients did not have any RAS mutation, and in these patients the overall survival was 25.8 months with panitumumab-FOLFOX4 versus 20.2 months for FOLFOX4 alone. By contrast, in the patients with only KRAS exon 2 mutations $(n=440)$, the overall survival was 15.5 months and 19.2 months-a better response with chemotherapy alone, which holds with previous findings. However, in the patients with unmutated KRAS but other $R A S$ mutations $(n=108)$, respective overall survival rates were 17.1 months and 17.8 months.

"We found these less-frequent $R A S$ mutations in $17 \%$ of patients who were initially considered nonmutant and these mutations were detrimental," says Douillard. "On the other hand, with more-stringent selection of patients without RAS mutations, the results of adding panitumumab to FOLFOX4 improved the efficacy in terms of both progression-free survival and overall survival."

In an accompanying commentary published alongside the report, Jordan Berlin from the VanderbiltIngram Cancer Center at Nashville, TN, who was not involved in the study, commented that these results "pose an intriguing challenge to medical oncologists, who now need to determine when, if ever, they will change their practices." Certainly, these findings need to be validated in other data sets from other trials. If validated, are we now at the stage where other defining features of colorectal cancer must be identified

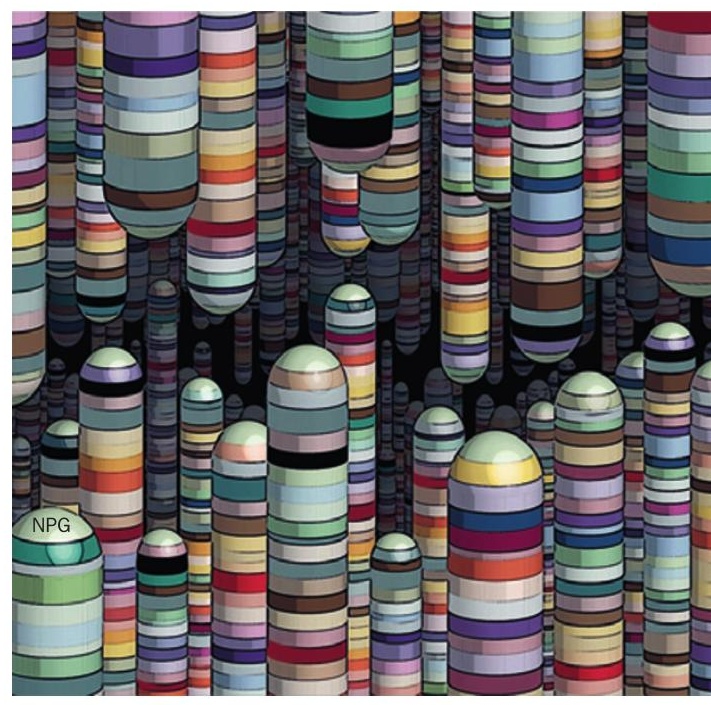

before patients are randomly assigned in clinical trials or are recommended treatment in the clinic? If patients with other RAS mutations actually perform worse with anti-EGFR therapies, simply ascertaining the KRAS exon 2 is not adequate to inform treatment.

"Indeed, on the basis of these results, the search for RAS mutations should not be restricted to KRAS exon 2, but extended to KRAS exons 3 and 4 -as well as NRAS exons 2, 3 and 4-to target patients and really apply the concept of personalized medicine," states Douillard.

Although these finding are important, additional mutations should also be studied along the Ras pathway, to better understand other mechanisms of resistance to therapy. "Ras is only one step in the cascade of activations along the pathway and should be studied in conjunction with pathways downstream," concludes Douillard.

Mina Razzak

Original article Douillard, J.-Y. et al. Panitumumab FOLFOX4 treatment as RAS mutations in colorectal cancer. N. Engl. J. Med. 369, 1023-1034 (2013) 